Volume: 1, Issue: 1, March 2016, Pages: 8-22, DOI: http://dx.doi.org/10.19082/ah8

\title{
MRI IMAGING AND PANCREATIC DISEASES
}

\author{
Mohammad Kazem Tarzamni ${ }^{1}$, Ali Kiani Nazarlou ${ }^{2}$ Jamil Abdolmohammadi $^{3}$
}

1: Department of Radiology, Imam Reza Teaching Hospital, Tabriz University of Medical Sciences, Tabriz, Iran

2: M.Sc. of Medical Imaging Technology, Department of Radiology, Imam Reza Medical Research \& Training Hospital, Golgasht Ave., Tabriz, Iran

3: M.Sc. of Medical Imaging Technology (MRI), Faculty of Paramedical Sciences, Kurdistan University of Medical

Sciences, Sanandaj, Iran

\section{TYPE OF ARTICLE: REVIEW ARTICLE}

\begin{abstract}
Diffusion Weighted Imaging (DWI) of the pancreas gland by apparent diffusion coefficient (ADC) map is important in the diagnosis and differentiation of diseases of the pancreas. The ADC value is affected by many factors, such as magnetic fields, motion, b-values, and types of pulse sequence. In this paper, the basic principles of diffusion magnetic resonance imaging (MRI) are discussed along with the use of this technique in the diagnosis of pancreatic diseases.

KEYWORDS: pancreas, diffusion-weighted magnetic resonance imaging, apparent diffusion coefficient
\end{abstract}

\section{INTRODUCTION}

Nuclear magnetic resonance has been used for more than 50 years as a chemical analysis technique, and, today, it is the basis of the MRI method. The word "nuclear" in "nuclear magnetic resonance" incorrectly implies that the technique has a nuclear nature or component, but that implication has been removed from the MR dictionary, and the term 'NMR tomography' has been replaced by "magnetic resonance imaging" (MRI) (1). MRI using a diffusion sequence has been used extensively as an imaging method for assessing intracranial diseases, and, recently, it has been used for investigating abdominal organs. In the past, due to its susceptibility to magnetic and motion artifacts, DWI provided inferior quality images in areas other than intracranial areas. Recently, due to technical advances in MRI, such as parallel imaging, multi-channel coils, and stronger diffusion gradients, this imaging technique is now used to investigate abdominal organs (2). Diffusion imaging takes images in which the diffusion rate of water molecules in intercellular spaces can be studied. The lower the diffusion rate (i.e., molecular motion) is in an area, the higher the intensity of the signals received from that area. Contrast material is not used in the technique (3). In this way, changes in the composition of tissue and the permeability of cells to the free movement of water are described by the apparent diffusion coefficient (ADC) (4). The intensities of the signals provided by diffusion weighted images are investigated qualitatively by visual observation and quantitatively by ADC measurements (2). Malignant tumors have high density cells and low extracellular volume (5). Among diagnostic imaging techniques, MRI is the method that provides information concerning anatomy and physiology with high resolution in some cases. This technique is performed without the use of ionizing radiation, and it shows the texture with high contrast (6). With regard to safety, efficiency, and high sensitivity of magnetic resonance imaging, valuable information can be obtained in the diagnosis of pancreatic disease, and this feature will be important for those who are at risk of diabetes

\section{FAMILIARITY WITH THE BASIC PRINCIPLES OF DIFFUSION MAGNETIC RESONANCE} IMAGING

2.1. What is Diffusion?

In diffusion imaging, the goal is to portray the movement of water molecules. Three types of molecule motion are as follows:

1) Bulk movement: Patients' movements during imaging leads to movement of the bulk water in the body. Therefore, the movement of water molecules in the bulk movement is greater than a pixel size, which

\section{Correspondence:}

Ali Kiani Nazarlou, Department of Radiology, Imam Reza Medical Research \& Training Hospital, Golgasht Ave., Tabriz, Iran. Tel: +989143140004, E-mail: Alikiani.n@gmail.com

Received: January 01, 2016, Accepted: February 28, 2016, Published: March 2016

iThenticate screening: February 28, 2016, English editing: March 02, 2016, Quality control: March 07, 2016

(C) 2016 The Authors. This is an open access article under the terms of the Creative Commons Attribution-NonCommercialNoDerivs License, which permits use and distribution in any medium, provided the original work is properly cited, the use is non-commercial and no modifications or adaptations are made. 
depends on the departure time and can have two different effects on the image. If that is the imaging time, results in impaired image (blurring and ghosting). If numerous pictures are produced, the images are notcoregistered together before and after movement. So, the bulk movement is seen in magnetic resonance imaging (7).

2) Flow: The movement is a water molecules direction, which causes a phase shift in the signal. Since the phase information is usually ignored, a small flow within a pixel cannot be seen (7).

3) Diffusion: The intra-voxel incoherent motion (IVIM), also called random motion or Brownian motion. This movement does not have any role in physiology; a water sample tube also can move around or it may even be stationary. If a drop of ink were spilled into a system, it will increase in size, while the center remains in the initial location, and the possibility of moving it along any arbitrary axis is the same. The Gaussian distribution of ink drop spreads (assuming the absence of obstacles) is what we want to measure (Figure 1) (7).

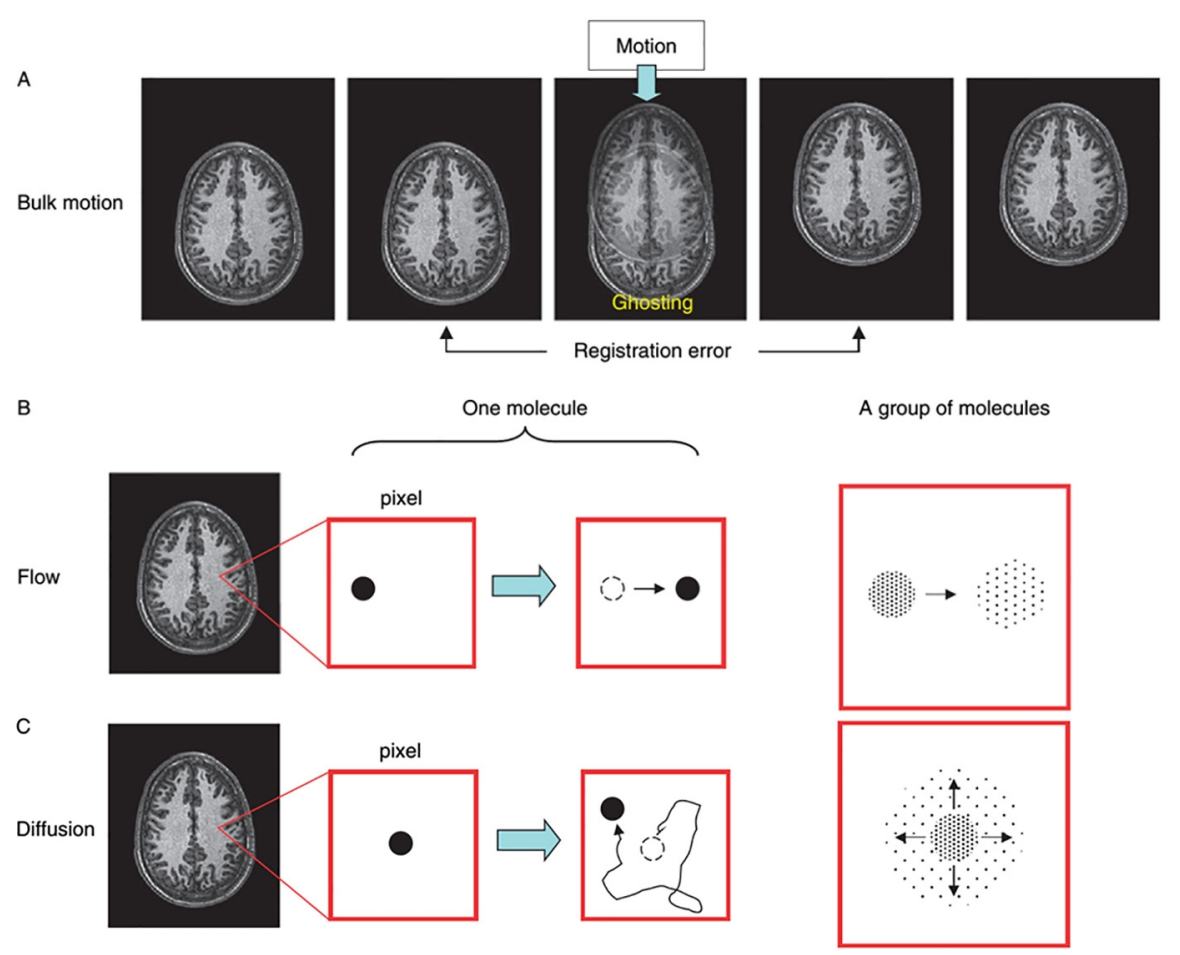

Figure 1. Difference between bulk motion (A), flow (B), and diffusion (C). The actual distance of water diffusion is approximately 5-10 $\mu \mathrm{m}$ during MR measurements, while the pixel size is typically 2-5 $\mathrm{mm}$. So, the water motion in (C) is exaggerated (7).

\subsection{How Is Diffusion Measured?}

As mentioned, usually in MR imaging, information concerning the intensity of the signal is determined primarily by the concentration of water (proton density). Also, the signal relaxation times, such as T1 and T2, affect the intensity of the signal. The goal in diffusion imaging is to make the intensity of the signal sensitive to the amount of water diffusion or diffusion constant. For this purpose, the pulsed magnetic field gradient is used, which is the technology that causes linear inhomogeneity of magnetic fields. In a magnet, the magnetic field called B0 isalong the magnet core in the $\mathrm{Z}$ direction. The $\mathrm{X}$ and $\mathrm{Y}$ axes are right-left and up-down orientations. When the $\mathrm{X}, \mathrm{Y}$, or $\mathrm{Z}$ gradients are used, the strength $\mathrm{B}_{0}$ is set linearly along each axis. We will control the power and the polarity of the gradient; in addition, the gradient can be turned on or off as desired. Gradients are usually turned on only for a short period of time $(1-100 \mathrm{~ms})$, hence they are called pulsed gradients (7). In MRI, the magnetic field $\left(\mathrm{B}_{0}\right)$ is kept as homogeneous as possible. When the gradient field is not applied, all water molecules have the same frequency (Figure 2A). Once the field gradient is applied, water molecules at different locations start to resonate at different frequencies (Figure 2B) (7). In Figure 3, the MR signal is shown after applying a pair of positive and negative gradients. After excitation RF pulse (time $t_{1}$ ), the protons on various occasions give MR signals with the same frequency. 
A

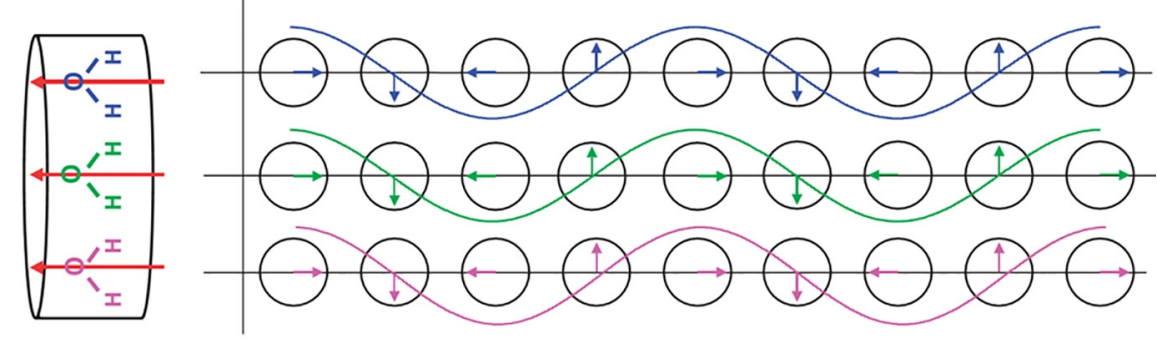

B

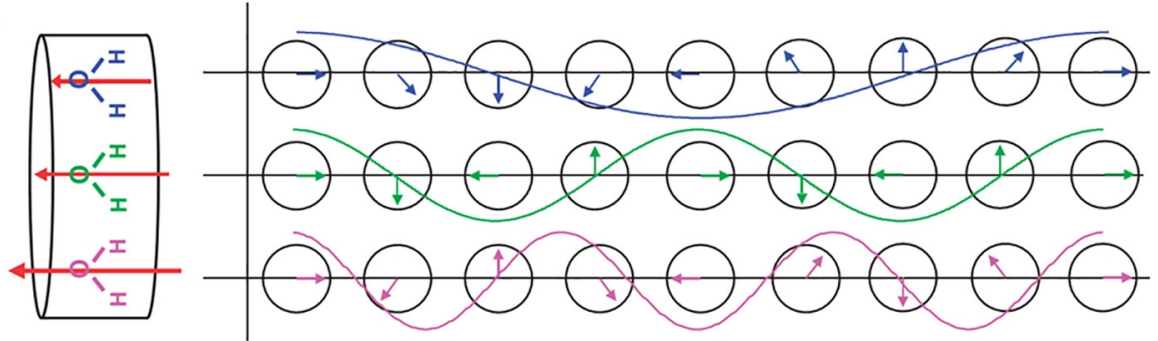

Figure 2. Schematic of field homogeneity and signal frequency: (A) Usually, the magnetic field (red arrows) is kept as homogeneous as possible to ensure that water molecules at different locations see the same field strength and give the same signal frequency; (B) When a gradient is applied, each water molecule is affected by a different field strength, depending on its location. In this example, pink water sees a stronger field and resonates at a higher frequency, while blue water resonates at a lower frequency (7).

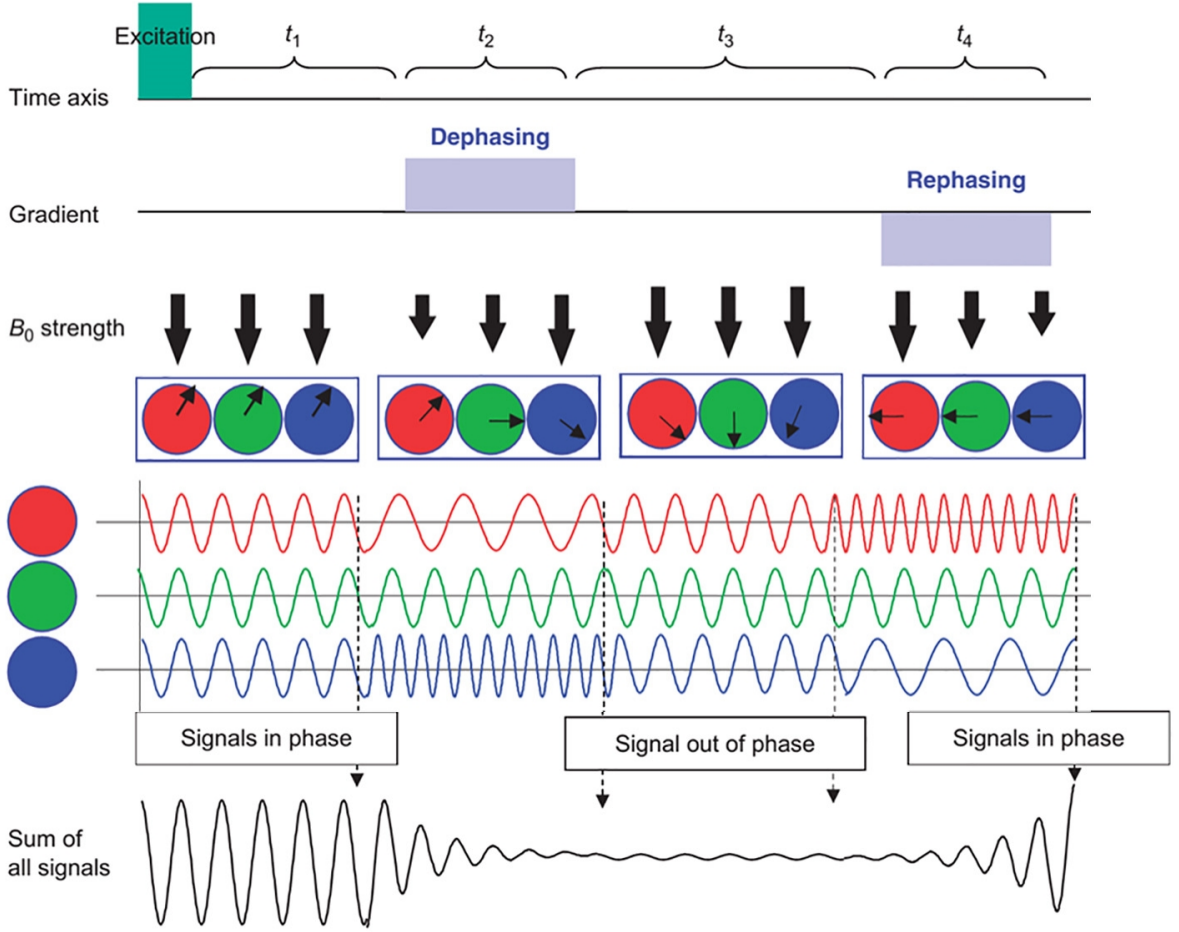

Figure 3. Example of a dephase-rephase experiment by gradient application: Red, green, and blue circles indicate three water molecules located at different positions in a sample tube. The thick arrows indicate the magnetic field strength $\left(\mathrm{B}_{0}\right)$, and the thin arrows indicate phases of the MR signals from each molecule (7).

During the first gradient application $\left(\mathrm{t}_{2}\right)$, the protons are subjected to different $\mathrm{B}_{0}$ and, depending on their positions, they resonate at different frequencies. In this example, the red proton was subjected to a weaker $\mathrm{B}_{0}$ and rotated slowly; the situation is reversed for the blue protons. At the end of gradient application and when the system regains 
the homogeneous $\mathrm{B}_{0}\left(\mathrm{t}_{3}\right)$, the signal phases (locations of the rotating arrows) of the protons are not equal. So, the first gradient is called the dephasing gradient. At time $t_{3}$, all protons resonate with the same frequency, while the phases of their signals still remain dephasing. At the time of the application of the second gradient $\left(t_{4}\right)$, which has opposite polarity from the first gradient, the red proton turns more quickly, and the blue proton turns more slowly. If the strength and duration of the application of the second gradient is the same as the first gradient, the protons get the same phase again at the end of the second gradient. Therefore, the second gradient is called the rephasing (7).

A

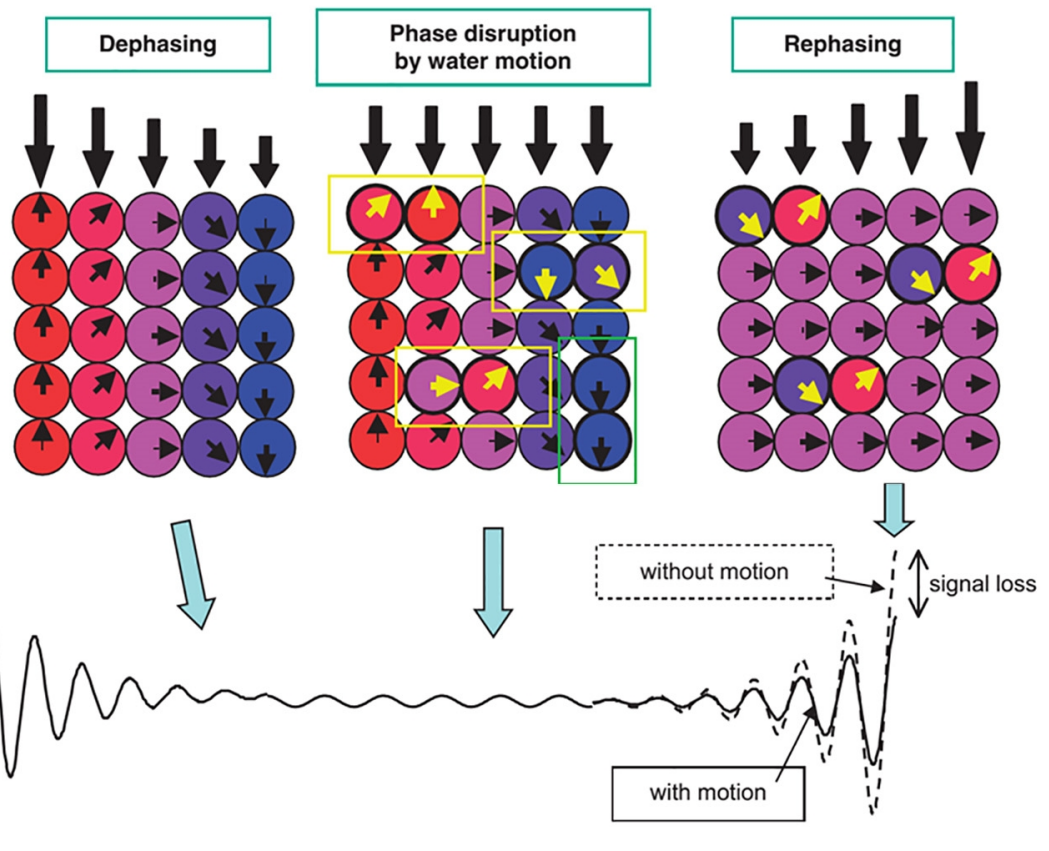

B

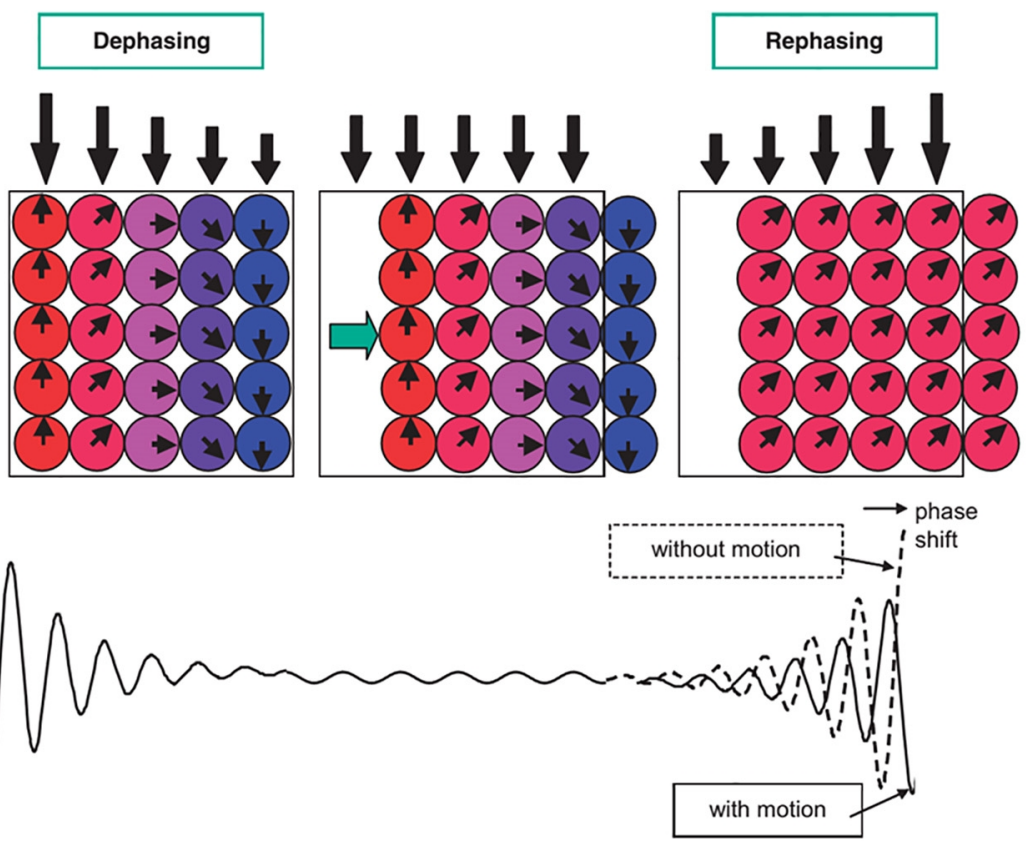

Figure 4. Effects of molecular motion in an experiment with a pair of gradients: The large black arrows indicate the strength of the magnetic field (B0), and the small arrows indicate phases of individual protons. The phases are also indicated by the gradation of colors: (A) Diffusion process in which protons move randomly. Protons (circles) that moved between the two gradients are indicated by boxes; (B) Coherent motion of water, such as flow or bulk motion (7). 
Although it seems that the gradients shown in Figure 3 do not do anything in practice, after their applications, the resulting signal is made sensitive to diffusion, so the signal becomes "diffusion weighted". In other words, the gradient "diffusion-weights" the signal. The rephasing is completed only when the locations of the water molecules do not change in the period between dephasing and rephasing gradient application. Figure 4A shows that the dephasing gradient sets the location of water molecules by their signals. If a water molecule moves, the phase gradient application is disrupted. After the rephasing gradient, we can identify the molecules that have moved because the phase of these molecules is different from the phase of the stable molecules. MRI cannot measure the phase of each water molecule, but it can show the rephasing done disrupted as a loss of signal (7).

\subsection{Important Point in Diffusion Measurement by MRI}

The diffusion imaging technique is non-invasive, and it does not require the injection of chemical tracers. It measures the movement of water molecules along the predetermined axis, and diffusion and flow motions lead to different outcomes in this experiment. As is shown in Figure 4B, coherent motions, such as flow and bulk, are entirely rephrased, which causes a phase shift of the signal (no signal loss), thus the incoherent (signal loss) and coherent motion (phase shift) can be distinguished in MRI diffusion measurement. Indiffusion measurement, the rate of molecules' motion in the time of measurement with respect to temperature, sample, and pulse sequence is 10-20 $\mu \mathrm{m}$, and the test is designed such that this rate of diffusion leads to a reduction in the signal in the range of10 to $90 \%$. In diffusion imaging, often the spin-echopulse sequence with unipolar gradients is used. To make the pulse sequence sensitive to the diffusion signal, bipolar gradients (positive and negative) can be used (Figure 5A).

A
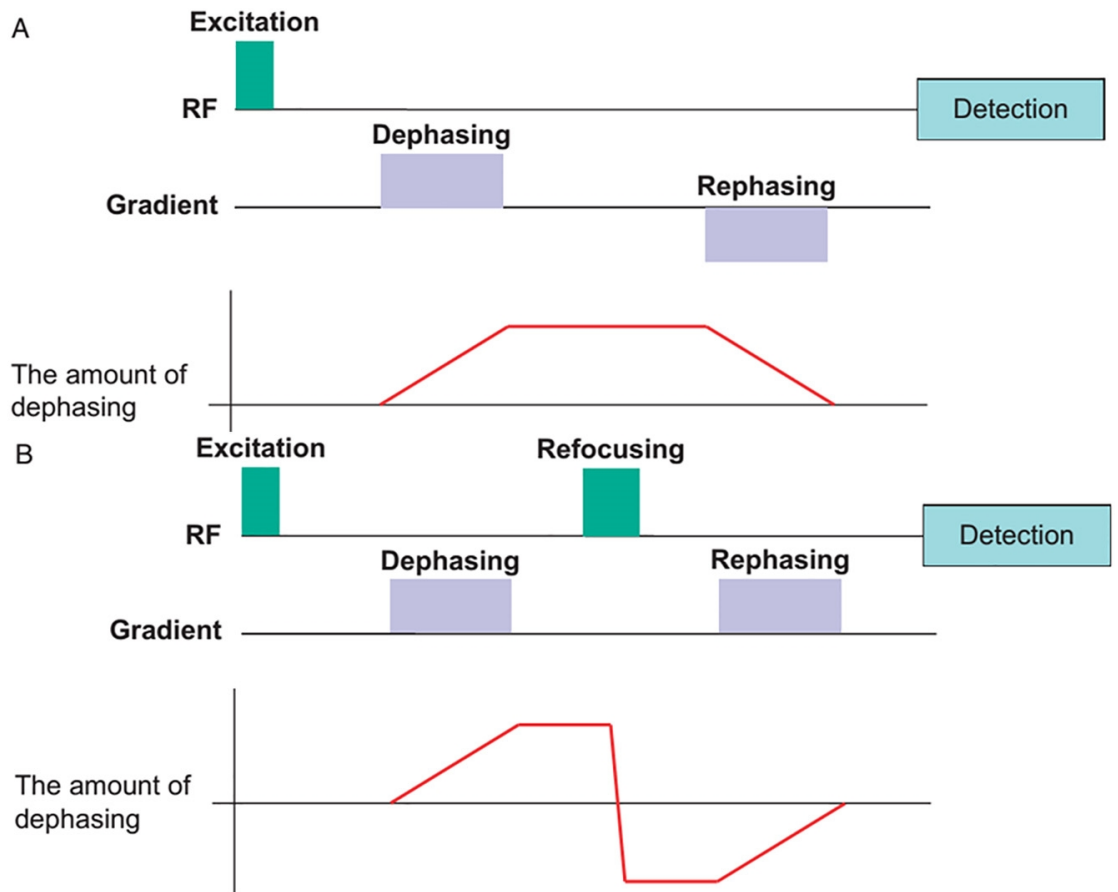

Figure 5. (A) Comparison of bipolar gradients; (B) comparison of unipolar gradients in a spin-echo sequence: Red lines show the amount of signal dephasing introduced by the first gradient (7).

A disadvantage of this method is a little reduction of the signal due to the effect ofT2 *decay in the time interval between two gradient pulses (typically20-40 ms). So, often, in order to refocus theT2 * decay, spin-echo pulse sequence in which the signal decay is duetoT2 effect and a little signal is lost in diffusion weighting signal is used. In this sequence, due to the use of rephasing pulse $180^{\circ}$, which reverses the signal phase, the rephasing and dephasing gradients are used in a unipolar way (Figure 5B). For both the unipolar and bipolar gradients methods, the experiment was made sensitive to the release of water molecules that dephased the signal phase. As stated earlier, the intensity of the MR signal is made sensitive to molecular diffusion by using the rephasing and dephasing gradients. Because the molecular motion in the time between the application of two gradient pulses causes incomplete rephasing, resulting in the reduction of the signal. If we consider the intensity of the signal without the 
application of diffusion gradients $\mathrm{S}_{0}$ and the intensity of the signal after the application of gradients $\mathrm{S}$, the factors that cause signal loss $\left(\mathrm{S} / \mathrm{S}_{0}\right)$ are as follows:

1) The time interval between the application of two gradient pulses $(\Delta)$ : by increasing the interval between the two pulses, the water molecules have more opportunities to move around, which causes a greater reduction of the signal.

2) Diffusion constant (D): increasing the diffusion constant increases the opportunities for water molecules to move around at a given time.

3) Initial dephasing amount: Another parameter is the amount of initial dephasing, which is decided by the area (strength $(\mathrm{G}) \times$ length $(\delta)$ ) of the gradients (Figure 6$)$.

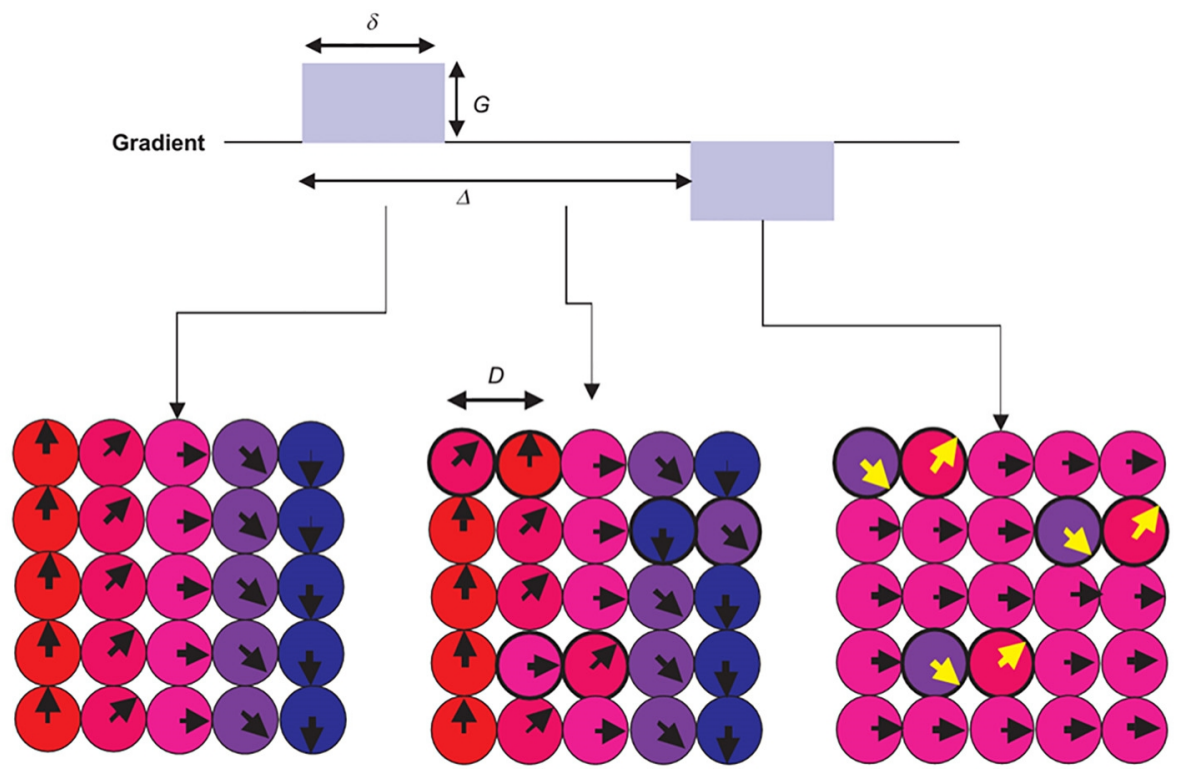

Figure 6. Parameters that affect the results of diffusion weighting (7).

Thus, four factors determine the amount of signal loss, i.e., $\mathrm{S} / \mathrm{S} 0=\mathrm{f}(\Delta, \delta, \mathrm{D}, \mathrm{G})$, where $\Delta, \delta$, and $\mathrm{G}$ are controllable factors, and the diffusion weight can be altered by changing them. The test results were signal intensities that are measurable with and without the application of diffusion gradients ( $\mathrm{S}$ and $\left.\mathrm{S}_{0}\right)$. Since $\Delta, \delta$, and $\mathrm{G}$ are specified, the diffusion constant (D) could be calculated. Thus, to observe signal loss, we must compare the results of two tests, one without the application of diffusion gradients (or weak) and one with the application of a stronger gradient. Changing the gradient separation $(\Delta)$ is not ideal because it changes the echo time and, thus, the resultant single loss would be influenced by both diffusion and $\mathrm{T}_{2}$ weighting (Figure $7 \mathrm{~B}$ ). Lengthening $\delta$ (Figure $7 \mathrm{C}$ ) works, but we usually do not have much room between the excitation and refocusing of RF pulses to accommodate longer $\delta$. The most common way to increase gradient strength $G$ is shown in Figure 7. In this way, we can keep the effects of proton density and relaxation times $\left(\mathrm{T}_{1}\right.$ and $\left.\mathrm{T}_{2}\right)$ unchanged, and the resultant signal loss would be solely due to the diffusion process (Figure 7) (7).

\subsection{Spin-echo Pulse Sequence Gradient PGSE: Pulsed Gradient Spin Echo}

The most common methods used for production of diffusion weighted contrast is the PGSE method and the Pulsed Gradient Spin Echo method. The sequence is created by a pair of RF pulses $90^{\circ}-180^{\circ}$ spin echo with large gradients on either side ofthe $180^{\circ}$ pulse. By manipulating the intensity of the gradient $(G)$, length $(\delta)$, and time interval between two pulses $(\Delta)$, we can determine the $\mathrm{b}$ factor (Figure 8). Diffusion weighted contrast (DW) treats fairly similar to $\mathrm{T} 2-\mathrm{W}$ that is inverse $\mathrm{T} 2$ in which the water-bearing tissues that have highly dynamic molecules, have lower signal intensity, while more solid and fixed tissues give a stronger signal. The strength of the signal is expressed by the following equation: $S(b)=S_{0} \exp (-b D)(1-1)$. In this equation, $S(b)$ is the value of the signal for a specific value of $b$. The value of $B$ depends directly on the intensity of the gradient $(G)$, the distance between the two lobes of the diffusion gradients $(\Delta)$, the duration of the gradient lobe $(\delta)$, and the diffusion constant of the tissue (D). In MRI, the apparent diffusion coefficient (ADC) is used. ADC can be calculated from two or more images with different $b$ values, and it can be shown as an ADC-map. A high D or ADC value is a sign of the high mobility of protons, so the signal will be turned on in a low DW-MRI and the corresponding ADC-map. Diffusion coefficient 
$\mathrm{D}$ has the units of $\mathrm{mm}^{2} / \mathrm{s}$. For example, at room temperature, pure water has a $\mathrm{D}$ value of approximately $2.2 \times 10^{-3}$ $\mathrm{mm}^{2} / \mathrm{s}$. With $\mathrm{b}$ equal to zero, the water signal has not been reduced, and the image will be T2-W; but with $\mathrm{b}$ equal to $1000 \mathrm{~mm}^{2} / \mathrm{s}$, the water signal will be reduced to $11 \%(9)$.

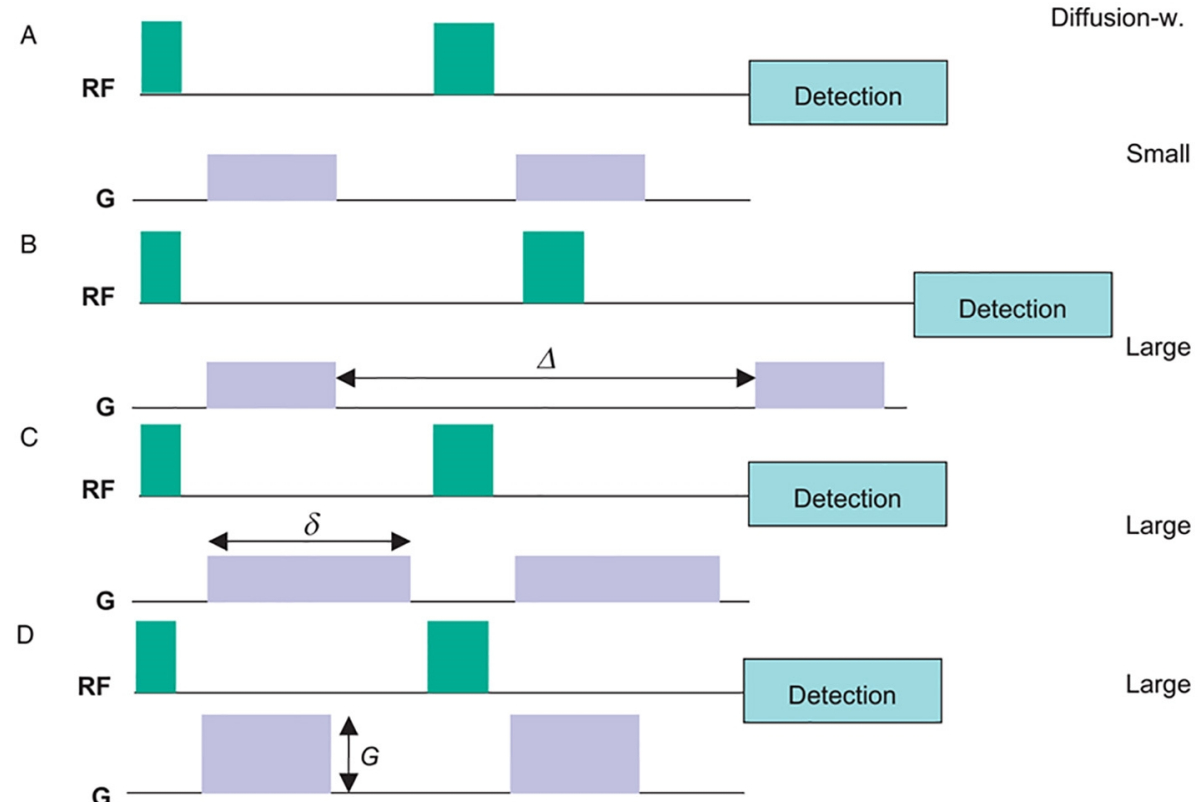

Figure 7. Several ways of achieving different diffusion weighting: Diagram (A) shows a spin-echo sequence with weak diffusion weighting. Diffusion weighting can be increased by lengthening $\Delta$ (B) or $\delta$ (C) or by applying a stronger gradient $G(D)$. Each experiment leads to different diffusion-weighting and T2 weighting (7).

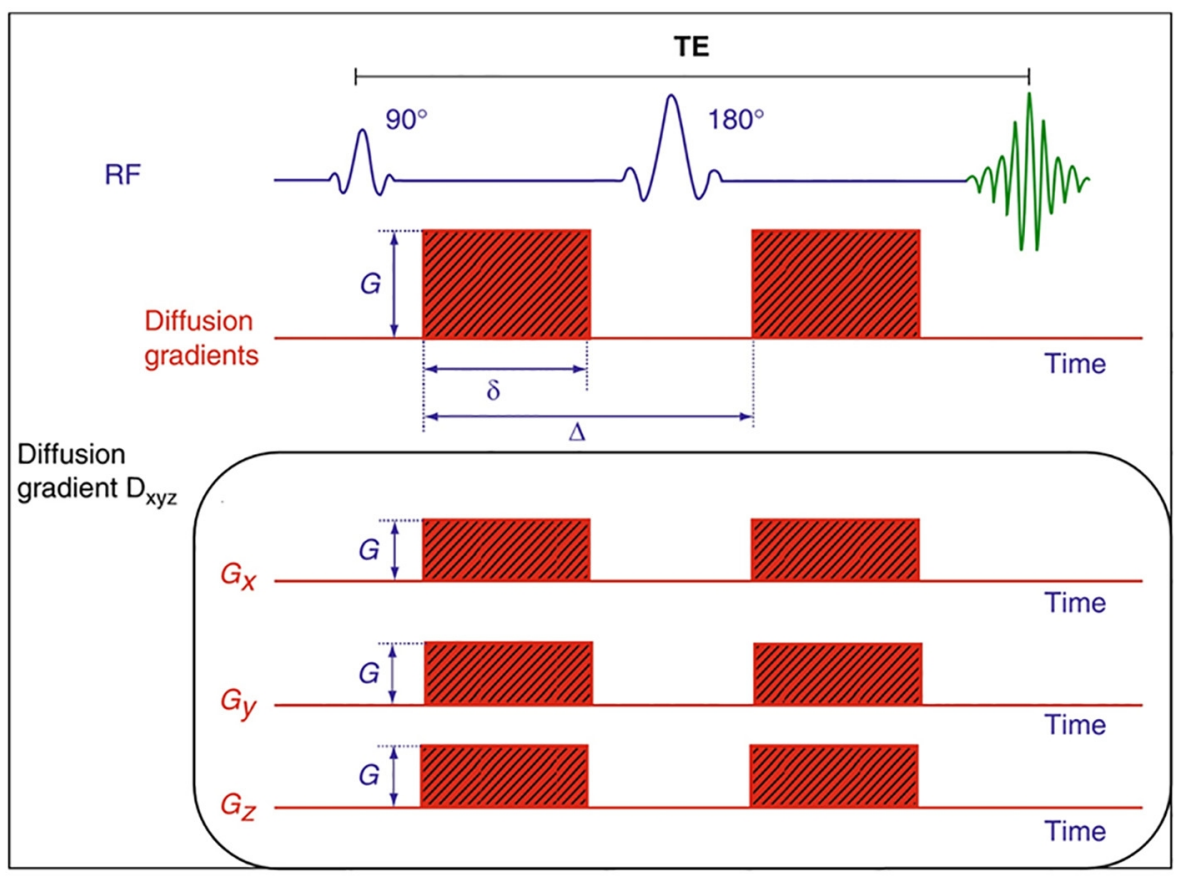

Figure 8. Pulse sequence PGSE sensitized to diffusion (8) 


\section{DWI AND DIRECTION-RELATED EFFECTS}

Diffusion gradients along all three axes can be applied individually or all three together. Separate accessibilities with different gradients make the sequence sensitive to limited release along a particular axis. This method is helpful in shooting areas that have different diffusion directions.

\subsection{DWI and Sequences}

In DWI, it is necessary to use Spin-echo sequences, since gradients should be applied to both sides of the RF pulse $180^{\circ}$ to make the sequence sensitive to changes in diffusion. In DWI, because even very little movement of a patient during imaging results in water movement in diffusion, rapid sequencing of this type of imaging is essential. Usually the very fast spin echotypes, such as SS-SE-EPI, are used, not because of rapid diffusion occurring, but because of the necessity for reducing other types of motion, such as flow, so that only the motion associated with diffusion is measured. Thus, typically, the Multi-shot SE-EPI and single are used to obtain images in a few seconds. So, artifacts induced by heart rate, respiration, and the patient's movements are decreased. However, conventional spin echo can be used in areas with lower motor artifacts. There are two types of image diffusion (18).

\subsection{Diffusion or Trace Images}

By applying diffusion gradients in the $\mathrm{x}, \mathrm{y}$, and $\mathrm{z}$ directions and combining images from each gradient function, we created a composite image of contrast-enhanced diffusion, which is called the trace weighted image (19).

\subsection{ADC Maps}

By calculating the ADC through post-processing for each voxel texture and giving a signal intensity consistent with its value, ADC maps will be obtained. Therefore, the limited tissue, which has a low ADC, is darker than free emission areas, which have high ADC, so that is unlike the trace images. ADC maps are helpful when shining in T2 shine-through has a problem. Brightness in T2 means T2's damages or areas with a very long T2 deficiency time on DW images or trace image remains light. So, it is difficult to know whether they represent a restricted diffusion area or not. It is possible to distinguish between a low ADC area and an area with long T2 deficiency time (7).

\section{THE USE OF DWI IN THE DIAGNOSIS OF PANCREATIC DISEASE 4.1. Diffusion Contrast Imaging of the Pancreas}

The use of DWI in the upper abdomen is increasing and this development in the clinical application of diffusion is mainly due to the improvement of the MRI technology, such as the development of stronger diffusion gradients and faster pulse sequences. Diffusion weighted images in the upper abdomen are made inrespiratoryarrestin20secondsor less. Like other areas of the body, the contrast in DWI in the upper abdominal area is mainly among the differences between the water molecules within the extra-and intracellular and vessel spaces. Diffusion refers to the random motion of microscopic water molecules in the body due to their kinetic energy, which leads to the net movement of water molecules. Free diffusion or isotropic diffusion occurs when the motion of the water molecules is completely random and in intracellular finite, and anisotropic diffusion occurs when the motion of water molecules is restricted due to the limitations of cell membranes and macromolecules. MRI measured signals in the body's water protons are more depending on whether they are in intracellular or extracellular spaces. In human organs, such as the pancreas, the motion of the water is not random; rather, it is limited by multiple tissue and cellular components, organelles, and membranes. In natural conditions, the limited movement of water is somewhat predictable, but it varies significantly among the organs. Various factors, such as the level of cells and perfused organs, determine the relative impedance of water diffusion. Generally, in tissues with low cellularity or in cells with ruptured membranes, water tends to move more than the tissue with relatively more cells (10). A conventional MRI sequence is not sensitive to random and microscopic motion of water molecules. However, the MRI signal can be made sensitive by adding a pair of balanced gradientsof magnetic field to water diffusion (11). The diffusion-sensitive gradients decrease the MR signal caused by the movement of water molecules (12). MR signals obtained with diffusion-sensitive gradients $\left(\mathrm{S}_{\mathrm{G}}\right)$ are achieved by the relationship among the gradients' signal intensities $(1-2): \mathrm{S}_{\mathrm{G}}=\mathrm{Se}^{-(\mathrm{b} \times \mathrm{ADC})}(1-2)$, where $\mathrm{S}$ represents the MR signal without a diffusion-sensitive gradient, $\mathrm{b}\left(\mathrm{s} / \mathrm{mm}^{2}\right)$ is determined based on the magnitude, duration, and time between the pair of diffusion gradients, and ADC is the ADC of water protons (12). The b-value reflects the sensitivity of the sequence to diffusion effects. In other words, as TE represents the $\mathrm{T}_{2}$ weighting, $\mathrm{b}$ value represents the diffusion-weighted values. We used the apparent diffusion coefficient term because the diffusion we measured indicated the contributions of the volume average of the diffusion of extra-and intracellular and vessel water protons from the voxel image. It should be noted that the increasing of factor $b$ in the equation $(2-$ 1) causes the loss of the MR signal in the constant ADC value. The b-factor value of $50 \mathrm{~s} / \mathrm{mm}^{2}$ is sensitive to large degree of motion or the much pure diffusion distance, while the b-factor value of $500 \mathrm{~s} / \mathrm{mm}^{2}$ is sensitive to low 
degrees of motion or to small pure diffusion distance. Similarly, the large ADC value of freely diffusible water molecules with a large degree of motion (e.g, intravascular space and fluid-rich structures in the body) will produce a greater loss in the MR signal than that for water molecules undergoing restricted diffusion (e.g, intracellular), which have a much smaller ADC value for a constant b-value $(13,14)$. Diffusion measurements with $b$ values less than $50 \mathrm{~s} / \mathrm{mm}^{2}$ are affected significantly due to the decrease in the MR signal resulting from perfused tissue $(15,16)$. Thus, in diffusion measurement in vivo, measurements shouldalways be performed with b-factor values greater than $50 \mathrm{~s} / \mathrm{mm}^{2}$ due to the isolation of the signal loss as a result of diffusion from perfusion. Due to the reduction of the signal-to-noise ratio with high values of factor $b$, the maximum $b$ value of $500 \mathrm{~s} / \mathrm{mm}^{2}$ is recommended for DWI of abdominal organs (17). Since molecular motions are small, usually DWI with TR repeat time and TE echo time is done with long $\mathrm{b}$ values to make the $\mathrm{b}$ value diffusion-sensitive to the MR signal. Thus, in addition to diffusion weight, DWI due to long TE time has substantial weight. In fact the intensity of the MR signal depends on both the $\mathrm{ADC}$ and the relaxation time of water in the imaging volume element that causes problems in the interpretation of DWI. Due to the combined weight of T2 and ADC, an area with a very long relaxation time (e.g, $400 \mathrm{~ms}$ ) may show a high signal in DWI while having a high $\mathrm{b}$ factor, and this can be confused with limited diffusion (13, 14). That effect is called $\mathrm{T}_{2}$ shine-through, which sometimes can be decreased by selecting a shorter TE or a higher $b$ factor, but the incidence of that in DWI is not easily avoided.

\subsection{Application of DWI in Pancreatic Cancer}

Oncologic applications of DWI are of interest in the diagnosis of pancreatic ductal adenocarcinoma because the lipophilic cell membrane must be considered. A tumor with dense cellular tissue functions as a barrier to free diffusion in extra-and intracellular spaces. Hence, the pancreas tumors should show limited diffusion compared to normal granular parenchyma on DWI (8). A normal pancreas has a higher mean ADC than a cancerous pancreas or a pancreas with mass-forming pancreatitis. Pancreatic cancer and pancreatitis are pancreatic lesions reported in medical clinics. In addition, proper and appropriate use of DWI may allow differentiation between the focal pancreatitis and ductal adenocarcinoma. For DWI acquired with $b=600 \mathrm{~s} / \mathrm{mm} 2$, mass-forming focal pancreatitis is virtually indistinguishable from the remaining pancreas (Figure 9), whereas pancreatic ductal adenocarcinoma tends to be hyper intense relative to the remaining pancreas (Figure 10).
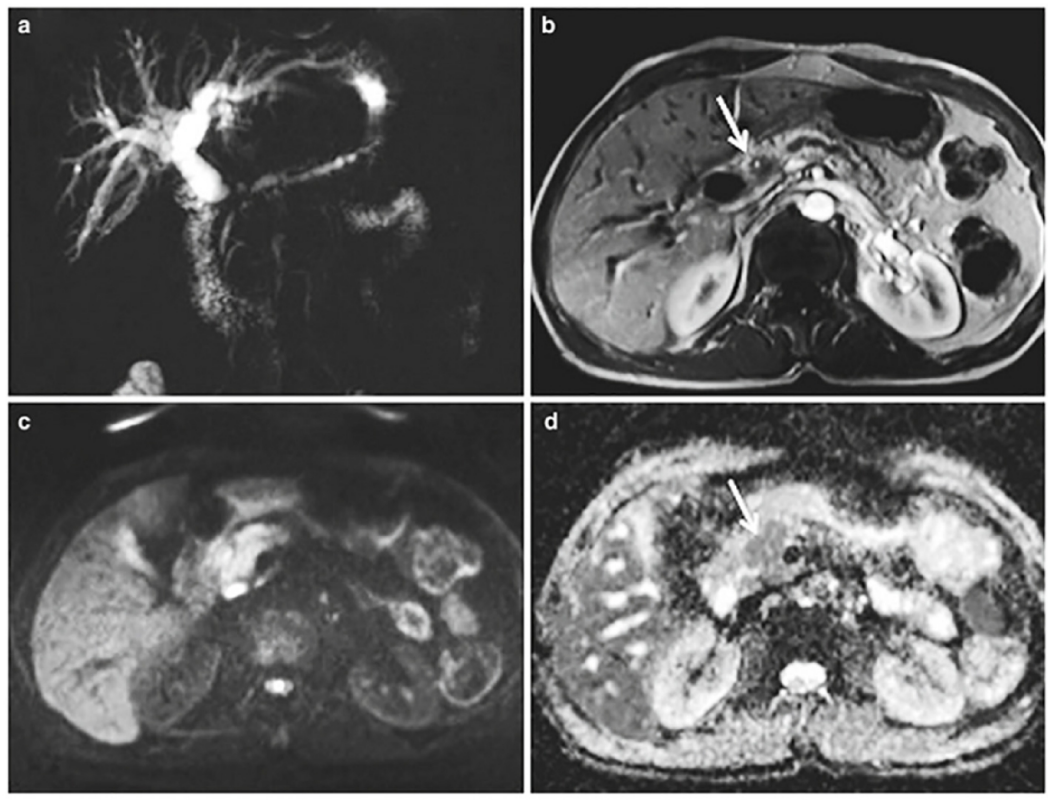

Figure 9. (a) Coronal-oblique thick section single-shot MRCP image shows a stricture of the biliary and pancreatic ducts, with the "penetrating duct" sign in the pancreatic duct; (b) Axial post contrast GE T1-weighted image with fat-suppression, obtained in the portal venous phase, showing a hypo vascular mass in the head of the pancreas (arrow); (c) DWI acquired with a b factor of $600 \mathrm{~s} / \mathrm{mm} 2$ shows the lesion with signal intensity that is the same of the pancreatic gland. The lesion demonstrates the same signal intensity as the pancreatic gland on the corresponding ADC map (d, arrow). These findings suggest mass-forming, focal pancreatitis (10). 
The mean ADC value of pancreatic adeno-carcinoma is significantly lower than normal pancreatic glandular parenchyma, whereas the ADC values of mass-forming focal pancreatitis and the normal pancreas do not differ significantly. DWI can be used in patients with suspected or known pancreatic cancer. First, as mentioned earlier, additional diffusion images are presented and more important information to characterize focal lesions can be identified by Computed Tomography (CT) or T1- or T2-weighted images and contrast-enhanced images. A focal lesion that shows limited diffusion is more likely to be malignant. Second, in patients with known cancer, the benefits of DWI include improved detection of peritoneal carcinomatosis and distant metastases. Because the free diffusion of water molecules due to the response and collapsing of tumor cells as treatment increases, the measuring of ADC values can be helpful as a quantitative tool for predicting and monitoring tumors' responses to treatment $(8$, $10)$.
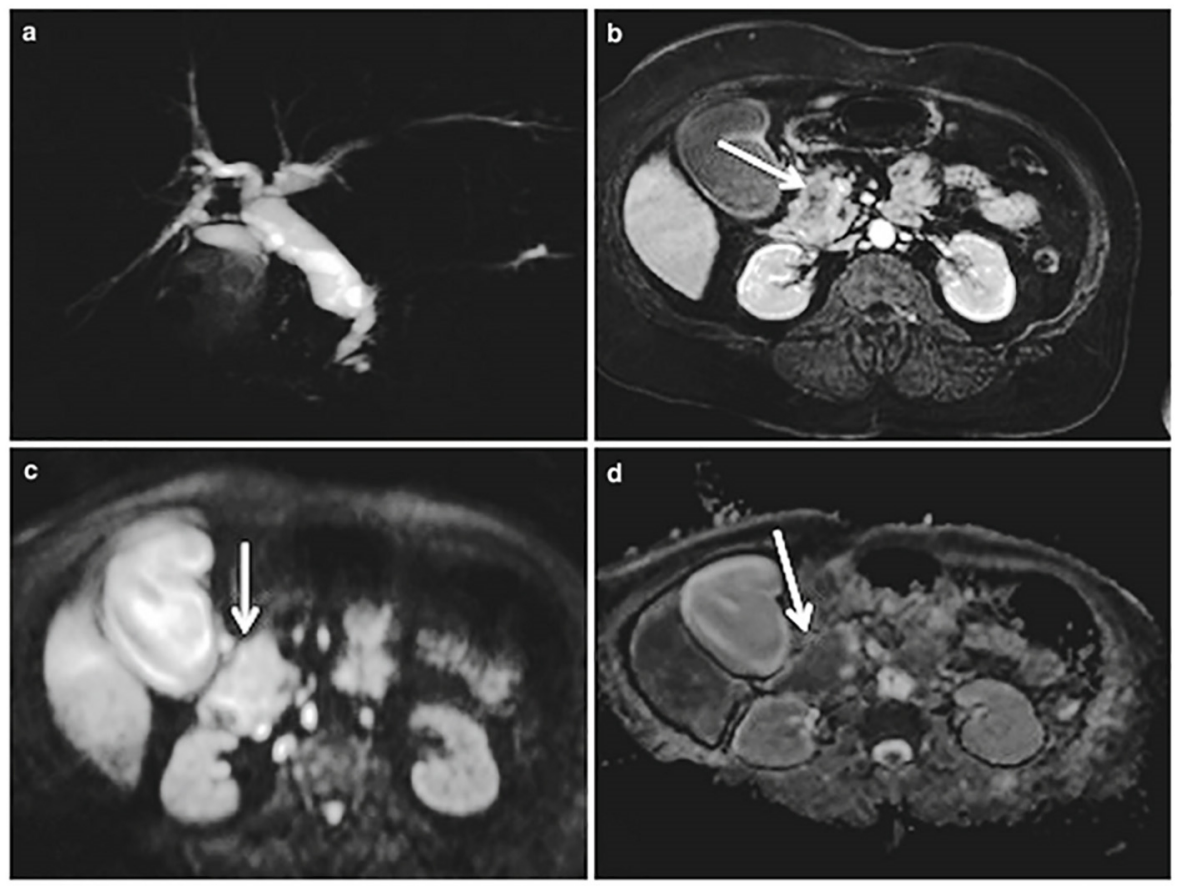

Figure 10. (a) Coronal-oblique thick section single-shot MRCP image shows an abrupt stenosis of the distal common bile duct, with proximal upstream dilatation. The pancreatic duct is normal. (b) Axial post contrast GE T1weighted image with fat suppression acquired in a late arterial phase shows a hypo vascular mass (arrow), which is causing the biliary ductal obstruction DWI acquired with a b factor of $600 \mathrm{~s} / \mathrm{mm} 2$; (c) shows the mass with high signal intensity and low signal intensity on the corresponding ADC map ( $\mathrm{d}$, arrows). This finding is characteristic of a malignant lesion. This case represents a histopathologically proven adenocarcinoma of the pancreas (10).

\subsection{Cystic Masses}

There is information that confirms that DWI can be used to identify and determine the significance of pancreatic cystic lesions (8). The observed signal intensities of all cystic lesions on DWI with lower b-values are high. However, when the factor b $1000 \mathrm{~s} / \mathrm{mm}^{2}$ applies, significant differences among the ratios of signal intensities of various cystic lesions of the pancreas are identified. In high b-value, the ratio of T2 shine-through to signal intensity decreases, and tissue cells will have more shares. At $b=1000 \mathrm{~s} / \mathrm{mm}^{2}$, the observed signals of simple cysts and pseudo-cysts of the pancreas are the isointense (Figures11 and 12). In contrast, the tumor cysts remain hyperintense (Figures 13-15). Thus, the high intensity of tumor cystson the images with $\mathrm{b}=1000 \mathrm{~s} / \mathrm{mm}^{2}$ cannot be attributed entirely to the influence of T2 shine-through. The ADC values are not affected by the T2 shine-through, and they are lower in the tumor cyst than in simple orpseudo-cysts. Hence, high signal on DWI due to diffusion is reduced that can be attributed to differences in inner content of cystic lesions. Since the cystic tumors have viscous contents, the $\mathrm{ADC}$ is decreased. In contrast, simple and pseudo-cysts have lower viscosity and therefore may increase the ADC $(8,10)$. 


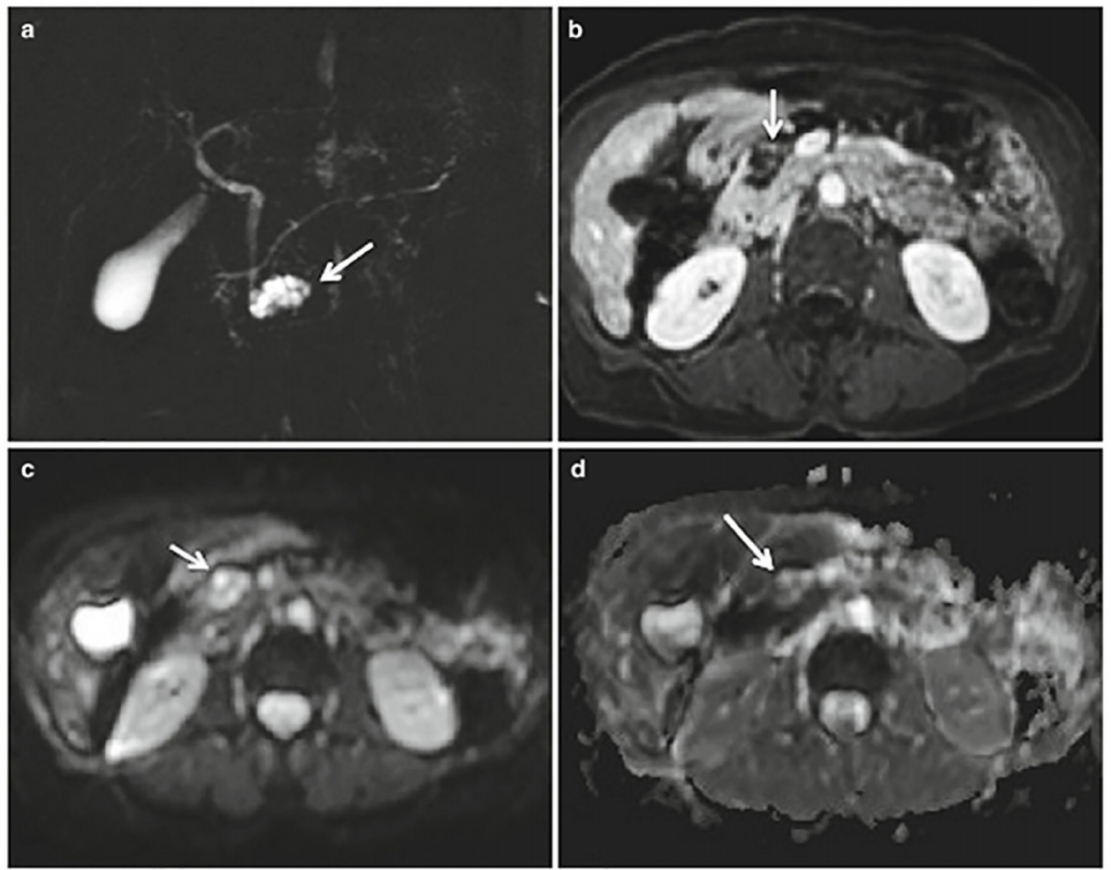

Figure 11. (a) Coronal-oblique thick section single-shot MRCP image; (b) Axial contrast-enhanced GE T1weighted sequence with fat suppression image: There is a multiloculated cystic lesion in the head of the pancreas, not communicating with the pancreatic duct. The high signal of the cystic lesion on both DWI with $b$ factor of 600 $\mathrm{s} / \mathrm{mm} 2$ (c) and on the corresponding ADC map (d, arrows) indicates lack of restricted diffusion, characteristic of a benign lesion (serous cystadenoma). Note also findings of divisum pancreas on the MRCP image (10).
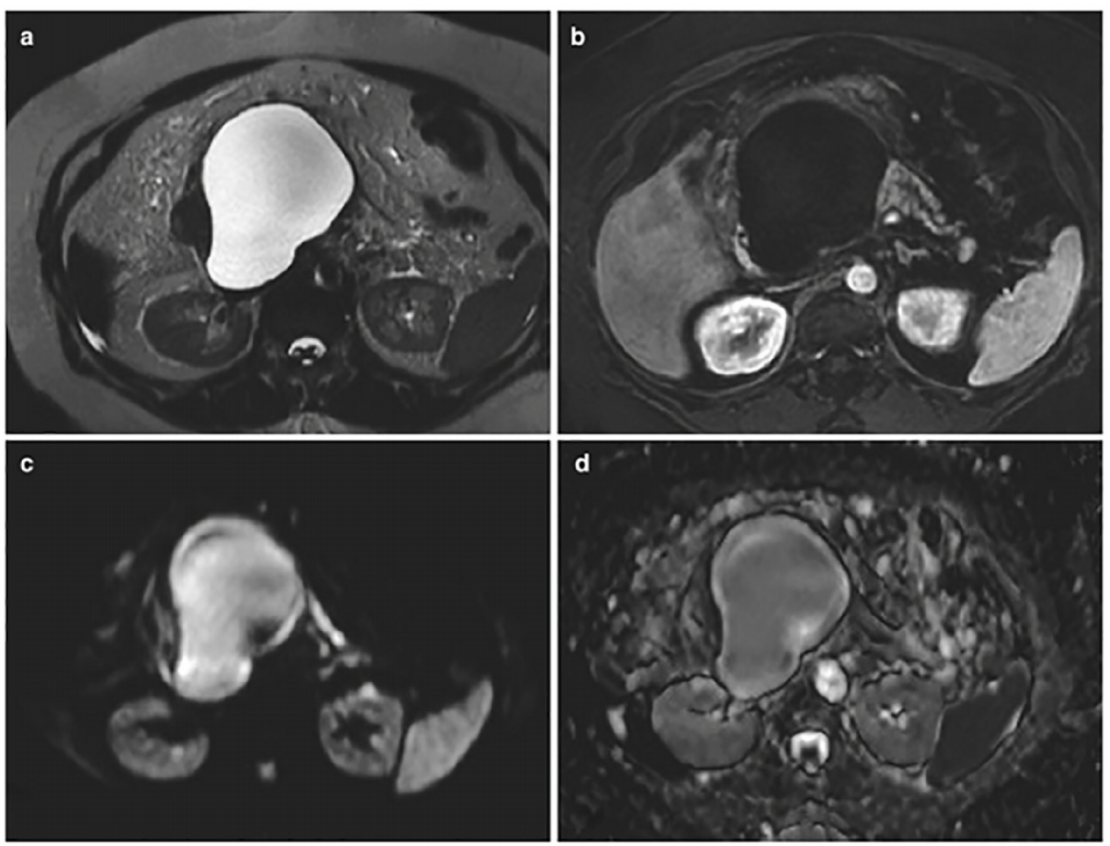

Figure 12. (a) Axial FSE T2-weighted image with fat suppression; (b) axial contrast-enhanced GE T1-weighted sequence with fat suppression shows a large unilocular cystic lesion in the neck of the pancreas: There is subtle enhancement of the wall of this cystic lesion; (c) The cystic lesion appears hyper intense compared with the pancreas in the DWI acquired with $b$ factor of $600 \mathrm{~s} / \mathrm{mm} 2$, as well as in the corresponding ADC map; (d)This finding is characteristic of a benign lesion (10). 

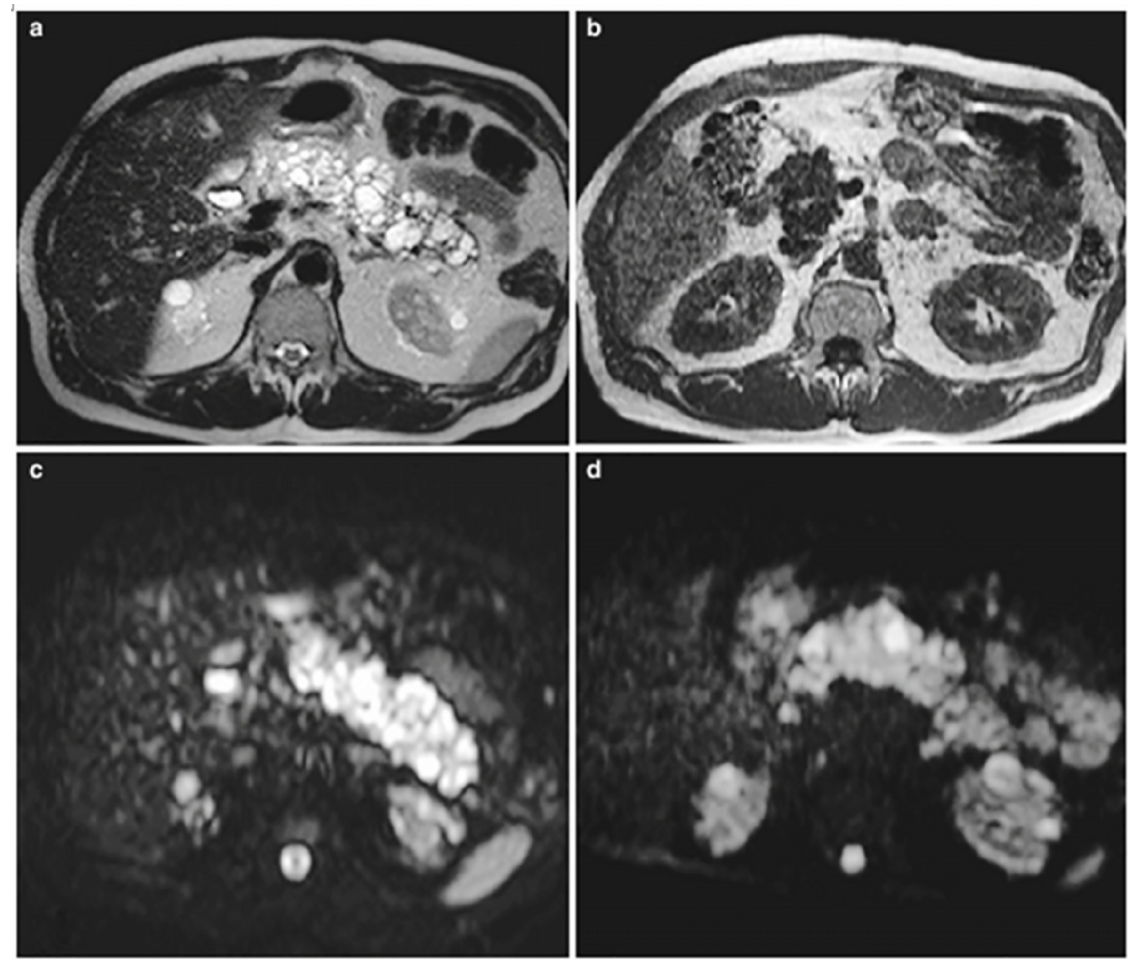

Figure 13. (a) Axial FSE T2-weighted image; (b) axial non-enhanced in-phase GE T1-weighted images show multiple small cysts throughout the whole pancreas. The cysts appear hyper intense on both diffusion-weighted images: (c) acquired with b factor of $0 \mathrm{~s} / \mathrm{mm}^{2}$; (d) acquired with b factor of $600 \mathrm{~s} / \mathrm{mm}^{2}(10)$.
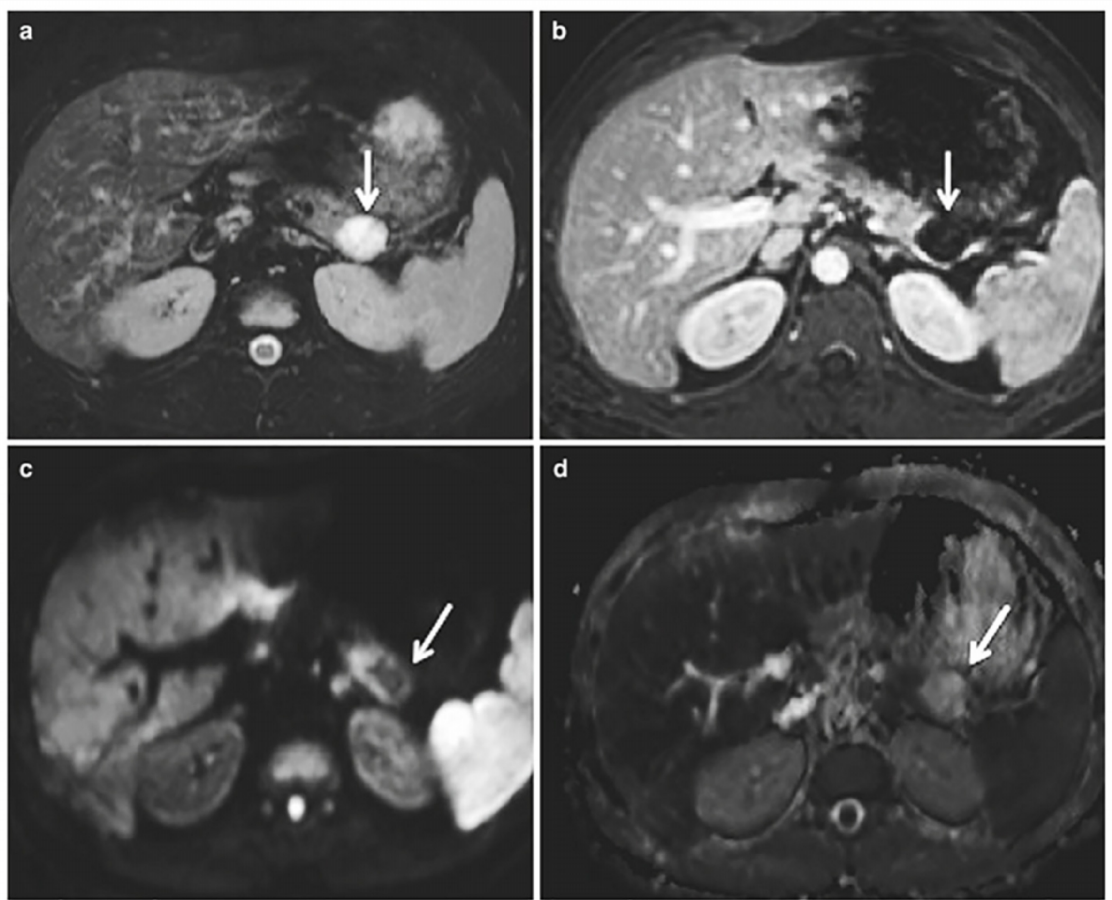

Figure 14. (a) Axial FSE T2-weighted with fat suppression; )b) axial postcontrast fat suppressed GE T1-weighted images show a cyst with a single enhancing septum and capsule. The lesion shows low central signal intensity in the DWI: (c) with b-value of $600 \mathrm{~s} / \mathrm{mm}^{2}$; (d) intermediate signal intensity on the corresponding ADC map: During surgery, the lesion was proven to be a mucinous cystadenoma, despite its slightly atypical appearance (10). 

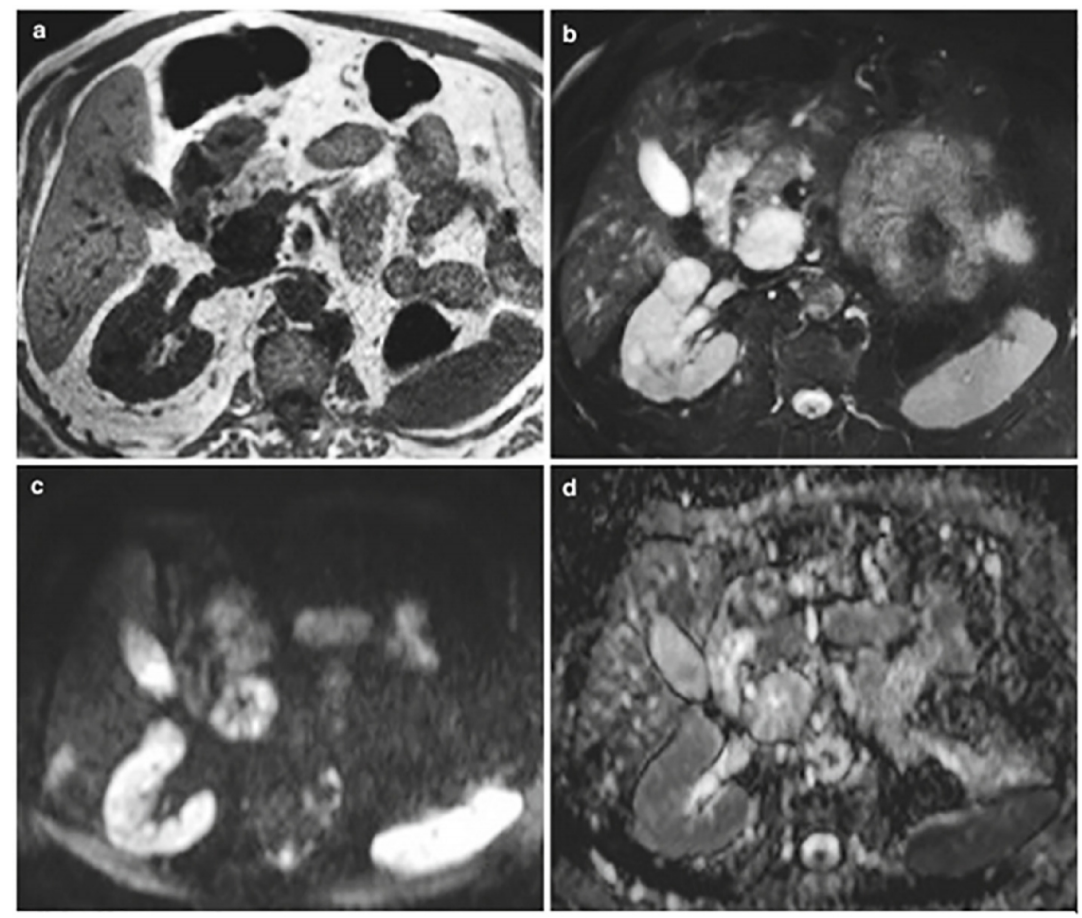

Figure 15. (a) Axial non-enhanced GE T1-weighted image; (b) axial fat-suppressed FSE T2-weighted image show a low and high (respectively) signal intensity mass in the uncinate process of the pancreas: (c) DWI acquired with $a b$ factor of $800 \mathrm{~s} / \mathrm{mm} 2$; (d) ADC map demonstrating the mass with high signal intensity in the DWI and low-tointermediate signal intensity in the corresponding ADC map (restricted diffusion) (10).
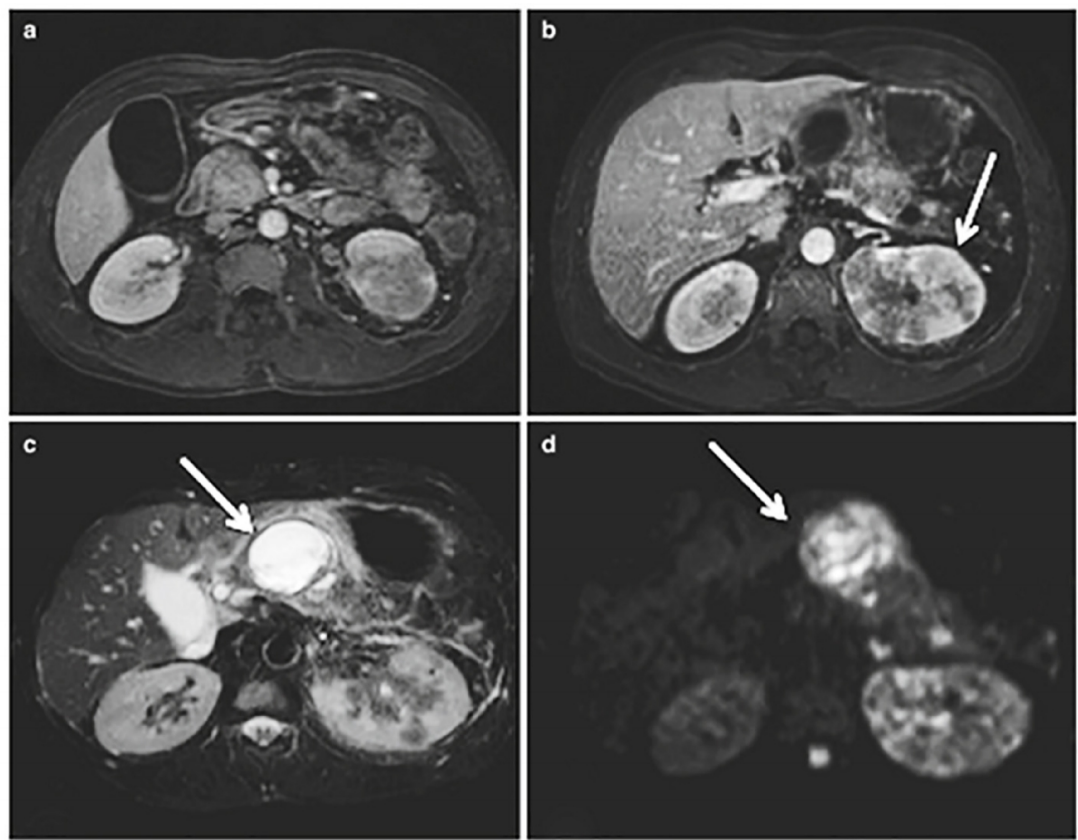

Figure 16. $(\mathrm{a}, \mathrm{b})$ Axial contrast-enhanced GE T1-weighted image with fat suppression showing an enlarged pancreas with heterogeneous enhancement and a peripancreatic fluid collection. The left kidney is enlarged and shows a striated pattern of enhancement, and there are associated inflammatory changes in the perinephritic fat (arrow); (c) Axial FSE T2-weighted image with fat suppression showing a hyperintense peripancreatic collection (arrow); (d) DWI with a $\mathrm{b}$ value of $1,000 \mathrm{~s} / \mathrm{mm} 2$ showing high signal intensity in the peripancreatic collection (arrow), the left kidney, and, to a lesser degree, in the pancreas (10). 


\subsection{Other Uses of DWI}

As in other abdominal organs, the use of DWI and ADC values have become of interest in the evaluation of pancreatic disease. In extreme cases, DWI increases the allowance of pancreatitis detection in a similar state of CT. Early data suggest that chronic pancreatitis effects the mobility of water molecules in the pancreas, such that patients with the disease demonstrate high signals on DWI (Figure 16). Furthermore, ADC values may correlate well with the severity of chronic pancreatitis, as assessed by the Cambridge classification. Finally, abnormalities seen on secretin-enhanced DWI may serve as an early predictor of pancreatic exocrine dysfunction, as after secretin stimulation, the ADC values reveal either delayed peak or lower peak values in patients with early chronic pancreatitis. Autoimmune pancreatitis (ALP) represents a distinct form of chronic pancreatitis that often presents as a pancreatic mass causing obstructive jaundice as well as pancreatic exocrine and endocrine insufficiency. It shows lower ADC values than chronic pancreatitis and pancreatic carcinoma. DWI also is useful in monitoring the effect of steroids during treatment (10).

\section{CONCLUSIONS}

Diffusion-weighted imaging (DWI) is a powerful, non-invasive imaging method that may yield useful qualitative and quantitative information about tumor cellularity and tissue structure. Publications exploring the use of DWI for evaluation of pancreatic disease are limited in number and scope. However, the value of DWI in various pancreatic conditions continues to be explored, and the available results suggest that there will likely be a niche for this technique in the clinic. DWI has been used successfully in body imaging as a complementary technique to the standard protocol or as a primary imaging tool. In pancreatic MRI, this technique can assess pancreatic neoplasm and pancreatic chronic inflammation in addition to standard imaging.

\section{ACKNOWLEDGMENTS:}

The authors thank the honorable managing director of the Tabesh Medical Center Imaging, Dr.M. H Abdkarimi, for their support.

\section{CONFLICT OF INTEREST:}

There is no conflict of interest to be declared.

\section{AUTHORS' CONTRIBUTIONS:}

All authors contributed to this project and article equally. All authors read and approved the final manuscript.

\section{REFERENCES:}

1) Hashemi RH, Bradley WG, Lisanti CJ. MRI: the basics. Lippincott Williams \& Wilkins; 2012.

2) Thoeny HC, De Keyzer F, Boesch C, Hermans R. Diffusion-weighted imaging of the parotid gland: Influence of the choice of $b$-values on the apparent diffusion coefficient value. J Magn Reson Imaging. 2004;20(5):786-90, doi: 10.1002/jmri.20196

3) Dale BM, Braithwaite AC, Boll DT, Merkle EM. Field strength and diffusion encoding technique affect the apparent diffusion coefficient measurements in diffusion-weighted imaging of the abdomen. Invest Radiol. 2009/12/23 ed. 2010;45(2):104-8, doi: 10.1097/RLI.0b013e3181c8ceac

4) Balci NC, Momtahen AJ, Akduman EI, Alkaade S, Bilgin M, Burton FR. Diffusion-weighted MRI of the pancreas: correlation with secretin endoscopic pancreatic function test (ePFT). Acad Radiol. 2008/09/16 ed. 2008;15(10):1264-8. doi: 10.1016/j.acra.2008.05.002, PMID: 18790398

5) SubratKumar R. Acute necrotising pancreatits: Current Concepts. Indian Journal of Surgery. 2005;67(2):78-86.. 2005;67(2).

6) Bammer R. Basic principles of diffusion-weighted imaging. European journal of radiology. 2003;45(3):169-84, doi: 10.1016/S0720-048X(02)00303-0

7) Mori S, Tournier J-D. Introduction to Diffusion Tensor Imaging 2e: And Higher Order Models. Academic Press; 2013.

8) Luna A, Ribes R, Soto JA. Diffusion MRI Outside the Brain. Springer; 2012.

9) Kartalis N, Lindholm TL, Aspelin P, Permert J, Albiin N. Diffusion-weighted magnetic resonance imaging of pancreas tumours. Eur Radiol. 2009 Aug;19(8):1981-90. doi: 10.1007/s00330-009-1384-8, PMID: 19308414

10) Soto JA, Castrillon GA, Anderson S, Holalkere N. Diffusion-Weighted MR Imaging of the Pancreas. Diffusion MRI Outside the Brain. Springer; 2012. p. 99-122. doi: 10.1007/978-3-642-21052-5_6, PMid: 22364342 
11) Le Bihan D, Breton E, Lallemand D, Grenier P, Cabanis E, Laval-Jeantet M. MR imaging of intravoxel incoherent motions: application to diffusion and perfusion in neurologic disorders. Radiology. 1986;161(2):401-7.; doi: 10.1148/radiology.161.2.37363909

12) Balci NC, Perman WH, Saglam S, Akisik F, Fattahi R, Bilgin M. Diffusion-weighted magnetic resonance imaging of the pancreas. Top Magn Reson Imaging. 2009;20(1):43-7.; doi: 10.1097/RMR.0b013e3181b48667

13) Padhani AR, Koh D-M, Collins DJ. Whole-body diffusion-weighted MR imaging in cancer: current status and research directions. Radiology. 2011;261(3):700-18.; doi: 10.1148/radiol.11110474

14) Koh D-M, Collins DJ. Diffusion-weighted MRI in the body: applications and challenges in oncology. Am J Roentgenol. 2007;188(6):1622-35; doi: 10.2214/AJR.06.1403

15) Baumann PS, Cammoun L, Conus P, Do KQ, Marquet P, Meskaldji D, et al. High b-value diffusionweighted imaging: A sensitive method to reveal white matter differences in schizophrenia. Psychiatry Res Neuroimaging. 2012;201(2):144-51; doi: 10.1016/j.pscychresns.2011.08.003

16) Colagrande S, Belli G, Politi LS, Mannelli L, Pasquinelli F, Villari N. The influence of diffusion-and relaxation-related factors on signal intensity: an introductive guide to magnetic resonance diffusionweighted imaging studies. J Comput Assist Tomogr. 2008;32(3):463-74; doi: 10.1097/RCT.0b013e318061961d

17) Takenaka D, Ohno Y, Matsumoto K, Aoyama N, Onishi Y, Koyama H, et al. Detection of bone metastases in non-small cell lung cancer patients: Comparison of whole-body diffusion-weighted imaging (DWI), whole-body MR imaging without and with DWI, whole-body FDG-PET/CT, and bone scintigraphy. J Magn Reson Imaging. 2009;30(2):298-308; doi: 10.1002/jmri.21858

18) Westbrook C, Roth CK. MRI in Practice. John Wiley \& Sons; 2013.

19) Smith MB. The Physics of Clinical MR Taught Through Images. Am J Roentgenol. Am Roentgen Ray Soc; 2010;194(4):W347-W347. doi: 10.2214/AJR.09.3190 